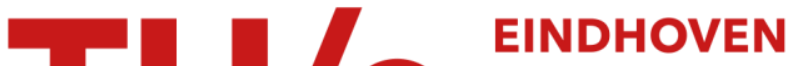 UNIVERSITY OF TECHNOLOGY
}

\section{Control Allocation for an Industrial High-Precision Transportation and Positioning System}

\section{Citation for published version (APA):}

Beerens, R., Thissen, S. C. N., Pancras, W. C. M., Gommans, T. M. P., van de Wouw, N., \& Heemels, W. P. M. H. (2021). Control Allocation for an Industrial High-Precision Transportation and Positioning System. IEEE Transactions on Control Systems Technology, 29(2), 876-883. [8947958]. https://doi.org/10.1109/TCST.2019.2956899

\section{Document license:}

TAVERNE

DOI:

10.1109/TCST.2019.2956899

Document status and date:

Published: 01/03/2021

\section{Document Version:}

Publisher's PDF, also known as Version of Record (includes final page, issue and volume numbers)

\section{Please check the document version of this publication:}

- A submitted manuscript is the version of the article upon submission and before peer-review. There can be important differences between the submitted version and the official published version of record. People interested in the research are advised to contact the author for the final version of the publication, or visit the $\mathrm{DOI}$ to the publisher's website.

- The final author version and the galley proof are versions of the publication after peer review.

- The final published version features the final layout of the paper including the volume, issue and page numbers.

Link to publication

\section{General rights}

Copyright and moral rights for the publications made accessible in the public portal are retained by the authors and/or other copyright owners and it is a condition of accessing publications that users recognise and abide by the legal requirements associated with these rights.

- Users may download and print one copy of any publication from the public portal for the purpose of private study or research.

- You may not further distribute the material or use it for any profit-making activity or commercial gain

- You may freely distribute the URL identifying the publication in the public portal.

If the publication is distributed under the terms of Article $25 \mathrm{fa}$ of the Dutch Copyright Act, indicated by the "Taverne" license above, please follow below link for the End User Agreement:

www.tue.nl/taverne

\section{Take down policy}

If you believe that this document breaches copyright please contact us at:

openaccess@tue.nl

providing details and we will investigate your claim. 


\title{
Control Allocation for an Industrial High-Precision Transportation and Positioning System
}

\author{
R. Beerens ${ }^{\circledR}$, S. C. N. Thissen, W. C. M. Pancras, T. M. P. Gommans, \\ N. van de Wouw ${ }^{\circledR}$, and W. P. M. H. Heemels ${ }^{\circledR}$, Fellow, IEEE
}

\begin{abstract}
We present a control allocation framework to improve the performance of an industrial high-precision translational transportation and positioning system based on an inverted permanent-magnet linear synchronous motor. Compared to the state-of-practice control solution, the proposed allocation technique achieves enhanced tracking performance and enlarged motion freedom, minimizes power consumption, and realizes relaxed hardware design specifications. A decentralized allocation algorithm is presented, which enhances the computational efficiency and facilitates the scalability to larger system configurations. The performance benefits of the proposed technique are illustrated by means of an experimental case study.
\end{abstract}

Index Terms - Control allocation, inverted permanent-magnet synchronous motor, motion control.

\section{INTRODUCTION}

$\mathbf{I}$ $\mathrm{N}$ THIS paper, we present a control allocation framework to improve the performance of industrial high-precision transportation and positioning systems (see Fig. 1), with respect to current state-of-practice control solutions. In particular, we consider a linear (i.e., translational) motion system, where multiple carriers can move on horizontal tracks, using the inverted permanent-magnet linear synchronous motor (IPMLSM) actuation principle, see [1]. In such a system, the coils of the three-phase actuators are located on the tracks, and the permanent magnets are placed on the carriers, see Fig. 1, such that the moving carriers do not have any electronics or cables attached to them. This principle makes the system highly suitable to be used in automated production lines involving, e.g., operation in vacuum, high temperature, or chemical environments. Due to these characteristics, such systems are widely used in industry in, e.g., the production of flat screens, OLED lighting, and photovoltaic solar cells [2].

Manuscript received June 20, 2019; revised November 13, 2019; accepted November 24, 2019. Date of publication January 1, 2020; date of current version February 9, 2021. Manuscript received in final form November 25, 2019. This work is part of the research programme CHAMeleon with project number 13896, which is (partly) financed by the Netherlands Organisation for Scientific Research (NWO). Recommended by Associate Editor S. Galeani. (Corresponding author: R. Beerens.)

R. Beerens and W. P. M. H. Heemels are with the Department of Mechanical Engineering, Eindhoven University of Technology, 5600 MB Eindhoven, The Netherlands (e-mail: r.beerens@tue.nl; w.p.m.h.heemels@tue.nl).

S. C. N. Thissen is with ASML, 5504 DR Veldhoven, The Netherlands (e-mail: stijn.thissen@asml.com).

W. C. M. Pancras is with MI Partners, 5507 TB Veldhoven, The Netherlands (e-mail: w.pancras@mi-partners.nl).

T. M. P. Gommans is with Microsure, 5692 EA Son, The Netherlands (email: t.m.p.gommans@tue.nl).

N. van de Wouw is with the Department of Mechanical Engineering, Eindhoven University of Technology, 5600 MB Eindhoven, The Netherlands, and also with the Department of Civil, Environmental and Geo-Engineering, University of Minnesota, Minneapolis, MN 55455 USA (e-mail: n.v.d.wouw@tue.nl)

Color versions of one or more of the figures in this article are available online at https://ieeexplore.ieee.org.

Digital Object Identifier 10.1109/TCST.2019.2956899

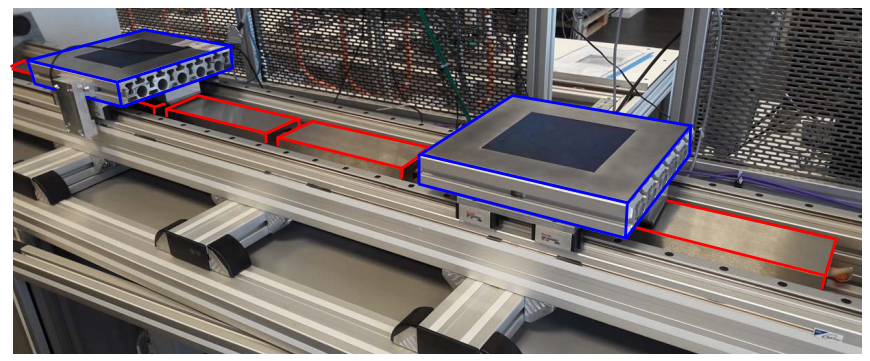

Fig. 1. Industrial IPMLSM-based transportation and positioning system. The carriers are indicated in blue, and the actuators are in red.

Multiple carriers, transporting (semi-finished) products, can move over fixed guidance tracks of arbitrary length in an automated production line. At specific locations along the production line, the carriers may be required to be positioned accurately, so that a certain operation on the product can be executed. At the same time, a subsequent carrier may move towards this operation stage and queues until the first carrier has moved on. This requires the system to allow for flexible, and independent motion of each carrier on arbitrary positions on the track. Furthermore, the system is typically over-actuated, since a carrier may commute with more than one set of coils in the track at the same time (i.e., one carrier is actuated by multiple actuators simultaneously), or multiple carriers may be influenced by the same set of coils (actuator) simultaneously. This may lead to conflicting control objectives for the actuators and results in large position errors or the inability to control carriers independently when using stateof-practice control solutions. Moreover, the actuator characteristics suffer from position dependence and end-effects (to be discussed in more detail in Section II) that pose design limitations in the sense that actuators must currently be placed at specific locations on the tracks. In this work, we aim to overcome the above-mentioned limitations by means of intelligent (allocation-based) control.

IPMLSM-based transportation systems are often controlled using field-oriented control (or vector control), see, e.g., [3], [4], where actuator redundancy is often handled through the use of commutation algorithms, see, e.g., [1], [5]. However, these existing commutation algorithms cannot handle independent control of multiple carriers simultaneously. Other control techniques that can handle actuator redundancy are, e.g., optimal control (see [6], [7]) or control allocation (see [7], [8]). Well-known examples of the former are linear quadratic control [6, Ch. 15], $\mathcal{H}_{\infty}$ control [6, Ch. 16-18], or model predictive control (MPC) [9]. For the current application, however, we choose to apply control allocation, since it offers several benefits over optimal control; it invokes a lower 


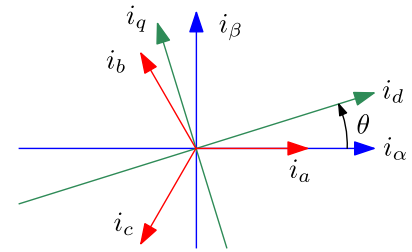

Fig. 2. Current reference frames for PMSMs.

computational effort [7], and, most importantly, separates controller design and tuning from the distribution of the resulting control efforts. In this way, well-known loop-shaping techniques (see, e.g., [10]), often used in industry for the tuning of motion controllers, can still be applied. Control allocation has been applied on a wide range of applications, e.g., on overactuated electric vehicles [11] and hard disk drives [12].

The main contributions of this paper are as follows. The first contribution is a control allocation framework for an IPMLSM-based transportation and positioning system. Compared to the state-of-practice control solution currently applied by the manufacturer, the proposed allocation technique achieves 1) improved tracking performance; 2) the possibility to perform-independent motion of multiple carriers; 3) minimization of power consumption; 4) a relaxation of hardware design rules; and 5) the possibility to take actuator limits into account. Second, we propose a decentralized control allocation approach that allows for real-time implementation of the proposed allocation scheme by significantly reducing the computational effort. The third contribution is an experimental case study on an industrial application. This paper builds upon our previous work in [13], which contains the controller design and a simulation example. In addition to [13], this paper contains a novel decentralized control allocation approach and an extensive experimental study.

The remainder of this paper is organized as follows. In Section II, we provide a detailed system description, and the proposed control allocation architecture is discussed in Section III. We illustrate the achievable performance benefits of the proposed controller by an experimental study in Section IV and provide conclusions in Section V.

\section{System DESCRIPTION}

This section starts with the basic actuation principle of an IPMLSM and the control challenges, followed by a model of the considered transportation system.

\section{A. Actuation Principle and Control Problems}

An inverted linear permanent-magnet synchronous motor has a magnet array attached to the carriers, and coils fixed to the tracks. In PMSM-based motion systems, the required three-phase currents to obtain the desired motion profile are typically generated in the so-called $d q 0$-reference frame (see [4, Ch. 6], [14, Ch. 10]) to simplify the control problem. Consider Fig. 2. The three-phase currents $i_{a}, i_{b}$, and $i_{c}$ (red) can be mapped onto the stationary $\alpha, \beta$-frame (blue) via the Clarke transformation [14, Ch. 10]. Next, the coil currents expressed in the $d q 0$-frame are obtained by the Park transformation $[14$, Ch. 10], i.e., by rotating the $\alpha, \beta$-frame by the commutation angle $\theta$. The coil currents are now expressed by the direct current $i_{d}$ and the quadrature current $i_{q}$ (green). For a translational system, this transformation results in a moving reference frame fixed to the carrier, where the $q$ direction is aligned with the direction of movement, by choosing the commutation angle in a specific way (depending on the carrier position). In this way, it can be realized that $i_{q}$ is the only a force-generating current by controlling $i_{d}$ to zero, such that only one input signal needs to be generated by a motion controller to achieve the desired motion of the carriers.

The considered inverted linear PMSM, however, suffers from end-effects. Due to the fact that the stator is segmented into groups of three-phase coils, there exist regions where the electronics in the tracks partially overlap a magnet array on a carrier. The correct commutation angle (i.e., such that $i_{q}$ indeed implements the desired control force, generated by the motion controller, on the carrier) is then a nonlinear function of the carrier position. The motor gain (i.e., the gain between the applied quadrature current and resulting force on the carrier) thus depends on the carrier position and the commutation angle.

The segmentation of coils also gives rise to the following control problems.

1) Given typical physical dimensions, carriers may be influenced by either one or two sets of coils (from now on referred to as "actuators"), leading to an over-actuated system.

2) Actuators may also influence multiple carriers at the same time. However, since an actuator is only able to implement a correct commutation angle (and thus a correct control force) for a single carrier, the other carrier experiences large disturbance forces as a result of the difference between the desired and attained control forces. This restricts the freedom in motion profile design, in the sense that two adjacent carriers cannot perform an independent motion accurately, as we will illustrate in Section IV.

3) Besides the aforementioned motion restrictions, an inverted linear PMSM suffers from hardware restrictions. The spacing between the actuators has to be specifically related to the length of the magnet array on the carrier to achieve a smooth transition of a carrier between two actuators, see Fig. 3 (where we assume that the magnet arrays have the same length as the carriers). That is, the center distance of two actuators must be equal to the length of the magnet array $L$. When using this particular spacing, the sum of the motor gains of the actuators that influence a given carrier is constant for all carrier positions. Then, if the same quadrature current $i_{q}$ is applied to multiple actuators, the applied force is independent of the carrier position. That is, there is no difference in the applied force to a carrier, regardless of whether the carrier is influenced by either one or two actuators. In this way, a single carrier may indeed be controlled accurately when actuated by multiple actuators (but does not allow 


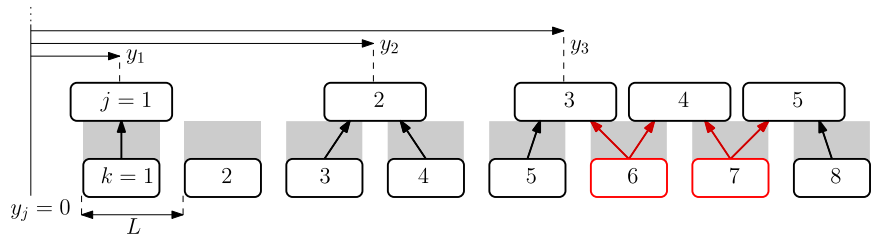

Fig. 3. Schematic representation of the IPMLSM-based transportation system. The region of influence of an actuator is marked in gray, and arrows denote the influence of an actuator (index $k$ ) on a carrier (index $j$ ). Actuators marked in red influence two carriers.

for the control of multiple neighboring carriers by the same actuator).

To address the above-mentioned limitations, we propose a control allocation framework in Section III that results in 1) enhanced tracking performance; 2) allowing independent motion of multiple carriers, despite the conflicting control objectives; 3) reduced power consumption; 4) relaxed hardware design specifications; and 5) the possibility to take actuator limitations into account.

\section{B. Carrier Transportation System Modeling}

Consider an IPMLSM-based carrier transportation system consisting of $n$ carriers and $m$ actuators, see Fig. 3. Let $j \in \bar{n}:=\{1,2, \ldots, n\}$ be the carrier number and $k \in$ $\bar{m}:=\{1,2, \ldots, m\}$ be the actuator number, used to uniquely identify all carriers and actuators in the system. The carrier transportation system is governed by the following dynamics:

$$
M \ddot{y}=B(y) u
$$

with $y=\left[y_{1}, \ldots, y_{n}\right]^{\top}$ the position of the carriers $\left(y_{j}=0\right.$ indicates the lower end of the tracks), and $M$ the diagonal mass matrix containing the carrier masses. We consider the $\alpha, \beta$-currents for each actuator as control inputs, instead of the frequently used $i_{q}$-current of the $d q 0$-frame. The essential motivation of using the fixed $\alpha, \beta$-frame is that the motor gain matrix $B(y)$ in (3) below is then independent of the commutation angle $\theta$. The motor gain matrix $B(y)$, hence, solely depends on the carrier positions, thereby simplifying the allocation problem in Section III. The input vector $u$ is then given by

$$
u=\left[i_{\alpha 1}, i_{\beta 1}, \ldots, i_{\alpha m}, i_{\beta m}\right]^{\top} .
$$

Due to the position dependence of the commutation between the carriers and the coils, the motor gain matrix is given by

$$
B(y)=\left[\begin{array}{ccccc}
b_{11}^{\alpha}\left(y_{1}\right) & b_{11}^{\beta}\left(y_{1}\right) & \ldots & b_{1 m}^{\alpha}\left(y_{1}\right) & b_{1 m}^{\beta}\left(y_{1}\right) \\
\vdots & \vdots & \ddots & \vdots & \vdots \\
b_{n 1}^{\alpha}\left(y_{n}\right) & b_{n 1}^{\beta}\left(y_{n}\right) & \ldots & b_{n m}^{\alpha}\left(y_{n}\right) & b_{n m}^{\beta}\left(y_{n}\right)
\end{array}\right]
$$

where $b_{j k}^{\alpha}$ and $b_{j k}^{\beta}$ are actuator-specific, position-dependent motor gains. The right-hand side of (1) then results in a column with forces applied on the carriers, i.e.,

$$
\tau=B(y) u=\left[\begin{array}{lll}
\sum_{k=1}^{m} \tau_{1 k} & \ldots & \sum_{k=1}^{m} \tau_{n k}
\end{array}\right]^{\top} .
$$

In (4), $\tau_{j k}$ is the force applied by actuator $k$ on carrier $j$, and is straightforwardly given by

$$
\tau_{j k}=b_{j k}^{\alpha}\left(y_{j}\right) i_{\alpha k}+b_{j k}^{\beta}\left(y_{j}\right) i_{\beta k} .
$$

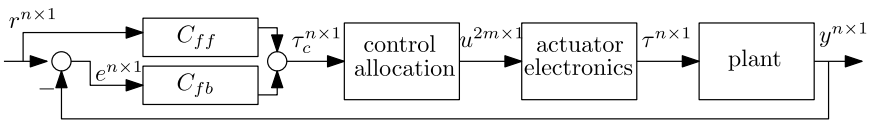

Fig. 4. High-level control architecture. $C_{f b}$ and $C_{f f}$ indicate the feedback and feedforward parts of the motion controller, respectively. The superscripts on the signals indicate their dimensions.

Note that some elements $\tau_{j k}$ in (4) may be zero if actuator $k$ does not influence carrier $j$, which is the case when the carrier is not close enough to the actuator in order to commute.

Let us introduce the relative position of carrier $j$ with respect to actuator $k$, i.e., $z_{j k}:=y_{j}-Y_{k}$, with $Y_{k}$ the position of actuator $k$ on the tracks, defined as the minimum position $y_{j}$ where actuator $k$ starts influencing a carrier $j$, i.e., $Y_{k}:=\min \left\{y_{j} \mid b_{j k}^{\alpha}\left(y_{j}\right) \neq 0 \vee b_{j k}^{\beta}\left(y_{j}\right) \neq 0\right\}$, which is independent of $j$. We are now ready to pose the following assumptions regarding the controllability of each carrier and similarity of the hardware components.

Assumption 1: $\operatorname{Rank}(B(y))=n$.

Assumption 2: All three-phase coil segments and permanent magnet arrays are identical.

As a result of Assumption 1, any carrier at any position on the tracks is influenced by at least one actuator, so that any desired $\tau_{j k}$ can be realized by the actuators. Assumption 2 is not necessarily needed for the developments in this paper but leads to significantly reduced complexity of $B$ and, therefore, easier implementation. A consequence of this assumption is that the motor gains of each actuator $k$ with respect to each carrier $j$ are identical. We can then simplify the motor gain matrix $B$ in (3) by writing $b_{j k}^{\alpha}$ and $b_{j k}^{\beta}$ in (3) as

$$
b_{j k}^{\alpha}\left(y_{j}\right)=b_{\alpha}\left(z_{j k}\right), \quad b_{j k}^{\beta}\left(y_{j}\right)=b_{\beta}\left(z_{j k}\right) .
$$

The motor gains in (6) are typically obtained from FEM-based electromagnetic simulations on the interaction between a carrier and an actuator, see [1]. By Assumption 2, we only have to perform these simulations for a single actuator/carrier interaction, thereby simplifying the implementation of the control allocation architecture below (where the gain matrix $B$ is explicitly used).

\section{Control Architecture}

We will first discuss the high-level control scheme and, subsequently, the allocation algorithm. Finally, we present a decomposition algorithm that enhances computational efficiency so that the control allocation architecture can be evaluated online at high sampling rates and facilitates implementation on large-scale system configurations.

\section{A. High-Level Control Scheme}

Consider Fig. 4. The reference signals $r$ (one for each carrier on the track) and error signals $e$ between the reference and the carrier positions $y$ are provided to a motion controller (composed of a feedback and feedforward part). This controller is typically designed using well-known loop-shaping techniques and generates the desired control forces $\tau_{c}=\left[\tau_{c 1}, \ldots, \tau_{c n}\right]^{\top}$. These desired control forces are then applied to the carriers by the AC actuators. Under the premise that the motion controller 
is stabilizing, which is the case by proper design, closed-loop stability is then preserved through control allocation. The primary objective of the control allocator is thus to find the currents $i_{\alpha k}$ and $i_{\beta k}$ in the control input $u$ in (2), such that the actuators indeed implement the desired control forces given by $\tau_{c}$. In other words, the actual forces acting on the carriers, denoted by $\tau$ in Fig. 4, should be equal to the desired control forces as determined by the motion controller, i.e., $\tau=B(y) u=\tau_{c}$. Due to the over-actuated nature of the system, the solution to the allocation problem $\tau=\tau_{c}$ (if attainable by the actuators) is not unique. We will exploit this freedom to introduce a second control objective, namely the minimization of power consumption by the actuators.

We will now discuss an unconstrained and a constrained allocation procedure. The latter incorporates a saturation constraint on the input, which is motivated by the desire from the industry to use cost-effective (less-powerful) actuators.

\section{B. Control Allocation}

We aim at implementing the desired control force generated by the motion controller on each carrier while minimizing the power consumption. The control allocation can then be described by the following convex quadratic programming (QP) problem [15, Ch. 4]:

$$
\min _{u} u^{\top} W u, \quad \text { s.t. } \tau_{c}=B(y) u
$$

where $u^{\top} W u$ is a quadratic metric for the power consumption, and $W$ is a symmetric weighting matrix. Since the optimization problem in (7) is convex and only contains one equality constraint, the explicit solution is given by [16]

$$
u=W^{-1} B^{\top}(y)\left(B(y) W^{-1} B^{\top}(y)\right)^{-1} \tau_{c} .
$$

In case there is a maximum allowable control input due to, e.g., the cost-effective hardware design, the criterion $\tau=\tau_{c}$ may not be attainable at all times. To deal with this scenario, we introduce a constraint on $u$ in the form of a limit on the current, and the difference between the desired and attained control forces $e_{\tau}=\tau_{c}-\tau$. This is then combined in an allocation problem that can be described by the convex, quadratically constrained quadratic program (QCQP) [15, Ch. 4] given by

$$
\begin{aligned}
& \min _{u, e_{\tau}} e_{\tau}^{\top} Q e_{\tau}+u^{\top} W u \\
& \text { s.t. } e_{\tau}=\tau_{c}-B(y) u \\
& \quad i_{\alpha k}^{2}+i_{\beta k}^{2} \leq i_{\max , k}^{2}, \quad \text { for all } k \in \bar{m}
\end{aligned}
$$

with $W$ and $Q$ symmetric weighting matrices, and $i_{\max , k}$ is the current limit on actuator $k$. The QCQP in (9) can be solved online using efficient algorithms, such as CPLEX [17] or Gurobi [18]. However, we propose a specific decomposition of the optimization problem in (9) to enhance computational efficiency. This favors solving (9) online at high sampling rates in large-scale industrial applications with many carriers and actuators. The proposed decomposition method also favors online computations of (8) in the unconstrained case.

Remark 1: Although the constraint in (9b) indeed resembles a constraint on the maximum current to be provided by the actuator, we can approximate the constraint by a set of polyhedral constraints. Then, the control problem reduces to a linear allocation problem [8], for which efficient QP algorithms are available, e.g., active-set or interior-point methods [19], which are studied in the context of control allocation in, e.g., [20] and [21].

Remark 2: We choose here to perform the control allocation in the $\alpha, \beta$-frame. In this way, we do not have to find an optimal commutation angle $\theta$ for each actuator, which would appear in the above-mentioned minimization problems as an extra decision variable when we would have chosen to apply the allocation in the $d q 0$-frame. Moreover, the motor gain matrix $B$ in (3) would then not only depend on the carrier position $y$, but on $\theta$ as well, making the equality constraint in (7) nonlinear. Performing the allocation in the $\alpha, \beta$-frame thus significantly simplifies the control allocation problem.

\section{Distributed Control Allocation}

To reduce computational costs (which favors real-time implementation on large-scale transportation systems), we decompose the allocation problems in (7) and (9) by 1) excluding actuators which do not influence any carrier from the allocation problem, and 2) solving multiple, less complex allocation problems subsequently, instead of the full allocation problem at once. The decomposition is done, at every sampling instant, by assigning carriers and actuators to specific subsets, and solving the optimization problem (7) or (9) for each subset separately. The only requirement for the proposed decomposition to apply is that the matrices $Q$ and $W$ are diagonal [to have decoupled costs in (7) and (9)]. By the dimensions of the carriers, each carrier is influenced by up to two actuators, whereas each actuator can influence up to two carriers. We first introduce the boolean variables $c_{j k} \in\{0,1\}$ given by

$$
c_{j k}= \begin{cases}1, & \text { when } b_{\alpha}\left(z_{j k}\right) \neq 0 \vee b_{\beta}\left(z_{j k}\right) \neq 0 \\ 0, & \text { otherwise }\end{cases}
$$

for any $j \in \bar{n}$ and $k \in \bar{m}$. In other words, $c_{j k}=1$ holds if and only if actuator $k$ influences carrier $j$.

Let us now discuss assigning the subsets of carriers. A carrier subset is given by $J_{p} \subseteq \bar{n}, p \in \bar{p}:=\{1,2, \ldots, P\}$ (where $p$ is the subset number and $P$ is the total number of subsets), and the related actuator subset is given by $K_{p} \subseteq \bar{m}$. Explaining the assignment of carriers and actuators to the subsets is best done by considering the problem in terms of a bipartite graph (see [22, Ch. 5]). Consider hereto Fig. 5. Let $G=(V, E)$ be a bipartite graph with $m+n$ vertices, consisting of $n$ carrier vertices $C_{1}, \ldots, C_{n}$, and $m$ actuator vertices $A_{1}, \ldots, A_{m}$ (see Fig. 5). Hence, $V=\left\{C_{1}, \ldots, C_{n}, A_{1}, \ldots, A_{m}\right\}$. The set of edges $E \subset V \times V$ is such that there is an edge between carrier vertex $C_{j}$ and an actuator vertex $A_{k}$ if and only if $c_{j k}=1$ [see (10)]. To explain our procedure to arrive at $J_{p}$ and $K_{p}$, $p \in \bar{p}$, we need the following definitions. A path (of length $l$ ) is given by $v_{1} v_{2} \ldots v_{l}$, where $\left(v_{i}, v_{i+1}\right) \in E, i=1, \ldots, l-1$ (in our case, this implies that a path consists of alternating carrier and actuator vertices). A subgraph $G^{\prime}=\left(V^{\prime}, E^{\prime}\right)$ of $G$ is a graph $V^{\prime} \subset V$ and $E^{\prime} \subset E$, where $E^{\prime}$ only consists of edges $\left(v, v^{\prime}\right)$ with both $v$ and $v^{\prime} \in E^{\prime}$. A (sub)graph is called connected if there is a path between all the vertices of the (sub)graph. With this terminology in place, we can now state the decomposition of the allocation problems (7) and (9). 


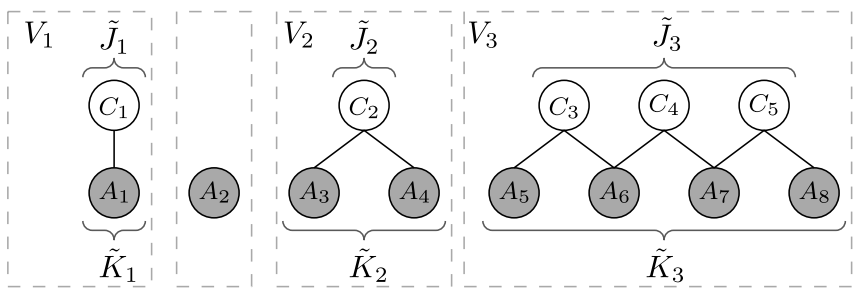

Fig. 5. Bipartite graph $G$ (corresponding to the schematic system representation in Fig. 3) with carrier vertices $C_{1}, \ldots, C_{5}$ (white) and actuator vertices $A_{1}, \ldots, A_{8}$ (gray). Its connected subgraphs $V_{1}, V_{2}$, and $V_{3}$ are indicated by dashed lines, where subgraphs containing only actuator vertices are discarded (hence, in this example, $P^{\prime}=4$ and $P=3$ ).

In our problem, we decompose the set $V$ now into its connected subgraphs with vertex sets $V_{p^{\prime}}, p^{\prime} \in \overline{p^{\prime}}:=\left\{1, \cdots, P^{\prime}\right\}$ (for which algorithms exist, see, e.g., [23, Ch. 6]). In case there are sets $V_{p^{\prime}}, p^{\prime} \in \bar{p}^{\prime}$, consisting only of actuators, we discard these sets such that we can exclude the associated actuators from the allocation problem, because these do not influence any carrier (i.e., the currents $i_{\alpha}$ and $i_{\beta}$ are zero for those particular actuators). A reordering then leads to the sets $V_{p}$, $p \in \bar{p}$, where $P \leq P^{\prime}$ because the sets in $V_{p^{\prime}}$ consisting only of actuator vertices are discarded, see Fig. 5. Due to physical properties, there are no sets in $V_{1}, \ldots, V_{P}$ that only consists of carrier vertices, as each carrier is influenced by at least one actuator, see Assumption 1.

We decompose the remaining sets $V_{p}, p \in \bar{p}$, as $V_{p}=$ $\tilde{J}_{p} \cup \tilde{K}_{p}$, with $\tilde{J}_{p}$ consisting only of carrier vertices and $\tilde{K}_{p}$ consisting only of actuator vertices, as indicated in Fig. 5. In this way, we obtain that the carriers are assigned to disjoint subsets $J_{p} \subseteq \bar{n}, p \in \bar{p}$, where $J_{p}$ contains the indices of the carriers contained in $\tilde{J}_{p}$, i.e., $\tilde{J}_{p}=\left\{C_{j} \mid j \in J_{p}\right\}$. Similarly, the actuators are assigned to disjoint subsets $K_{p} \subseteq \bar{m}$, $p=1,2, \ldots, P$. Clearly, $J_{p}$ and $K_{p}$ are related in the sense that the carriers in $J_{p}$ are only influenced by actuators in $K_{p}$ (and not by any others, see Figs. 3 and 5). Moreover, an actuator in $K_{p}$ has an influence on at least one carrier in $J_{p}$, i.e., if $k \in K_{p}$ there is $j \in J_{p}$ such that $c_{j k}=1$. We have that $J_{p} \cap J_{p^{\prime}}=\emptyset$ when $p \neq p^{\prime}$ and $\bigcup_{p=1}^{P} J_{p}=\bar{n}$ by Assumption 1 (i.e., $\left\{J_{1}, \ldots, J_{P}\right\}$ forms a partition of $\bar{n}$ ). Also, $K_{p} \cap K_{p^{\prime}}=\emptyset$ when $p \neq p^{\prime}$ and $\bigcup_{p=1}^{P} K_{p} \subset \bar{m}$, and thus, $\left\{K_{1}, \ldots, K_{P}\right\}$ forms a partition of $\left\{k \in \bar{m} \mid c_{j k}=1\right.$ for some $\left.j \in \bar{n}\right\}$. Hence, actuators that do not influence any carrier are not in $\bigcup_{p=1}^{P} K_{p}$. As a result, these actuators are excluded from the allocation problem, improving computational efficiency. Note that two carriers are in the same set $J_{p}$ if and only if there is a connection (path) via multiple actuators/carriers between them (see Fig. 5) and, hence, in the allocation problem, these have to be treated simultaneously. Sets $J_{p}$ (combined with $K_{p}$ ) and $J_{p^{\prime}}$ (combined with $K_{p^{\prime}}$ ) for $p \neq p^{\prime}$ can be considered separately in the allocation problem as there is no path between any of the elements in $J_{p}$ (and $K_{p}$ ) and $J_{p^{\prime}}$ (and $K_{p^{\prime}}$ ).

Algorithms are available to decompose a graph in its connected subgraphs (see, e.g., [23, Ch. 6]). However, in our case, we obtain the sets $J_{p}$ and $K_{p}, p \in \bar{p}$ by exploiting a physical ordering of the carriers and actuators (without loss of generality): the carriers and actuators are logically numbered 1 to $n$ from left to right on the tracks (see Fig. 3).

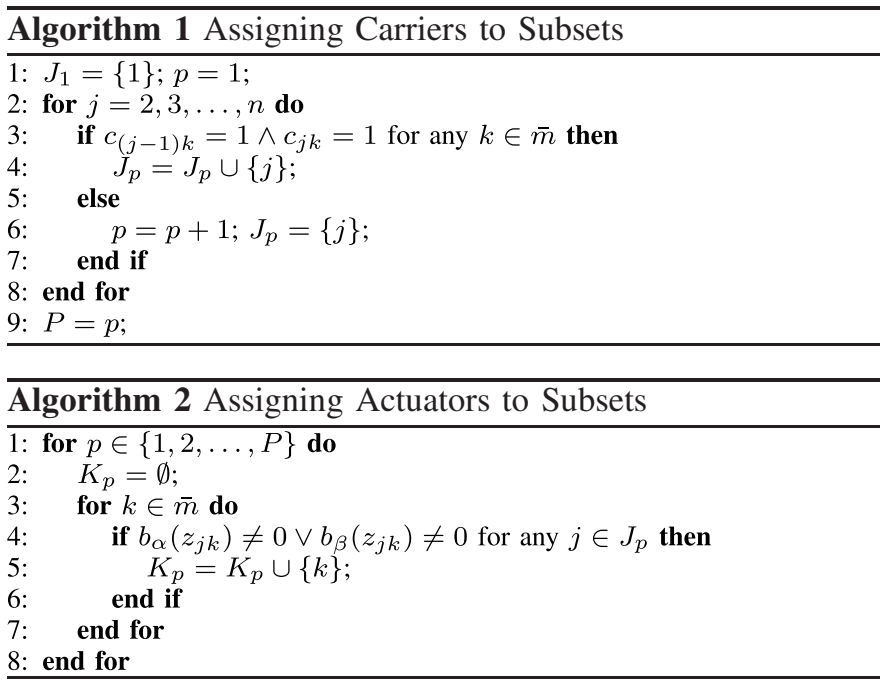

Moreover, using the fact that a carrier is influenced by either one or two carriers and each actuator influences at most two carriers, we can simplify the generic algorithms typically used to decompose a graph into its connected subgraphs (see [23, Ch. 6]), leading to Algorithms 1 and 2. We use these algorithms in the experimental results in Section IV.

As a result of the decomposition, the solution for $u$ in (8) in the unconstrained allocation problem (7) changes to the following set of solutions:

$$
u^{p}=W_{p}^{-1} B_{p}^{\top}\left(y_{p}\right)\left(B_{p}\left(y_{p}\right) W_{p}^{-1} B_{p}^{\top}\left(y_{p}\right)\right)^{-1} \tau_{c}^{p}
$$

for all $p \in \bar{p}$, where $y^{p}$ and $\tau_{c}^{p}$ are the positions of the carriers and the desired control forces in subset $J_{p}$, respectively. Moreover, $B_{p}$ and $W_{p}$ correspond to the submatrices of the motor gain matrix $B$ and the (diagonal) weighting matrix $W$, respectively, corresponding to $K_{p}$. Using this decomposition method, the computational effort [in the sense of computing the inverse in (8)] is significantly reduced due to the smaller matrix $B_{p}$, compared to $B$. The constrained optimization problem in (9) now changes to a set of problems that are solved subsequently for all $p \in \bar{p}$ :

$$
\begin{aligned}
\min _{u^{p}, e_{\tau}^{p}} & e_{\tau}^{p \top} Q_{p} e_{\tau}^{p}+u^{p \top} W_{p} u^{p} \\
\text { s.t. } & e_{\tau}^{p}=\tau_{c}^{p}-B_{p}\left(y^{p}\right) u^{p} \\
& i_{\alpha k}^{2}+i_{\beta k}^{2} \leq i_{\max , k}^{2}, \quad \text { for all } k \in K_{p}
\end{aligned}
$$

where $Q_{p}$ corresponds to the submatrix of the (diagonal) matrix $Q$ corresponding to $J_{p}$. The decomposed optimization problem (12) contains less decision variables compared to its nominal counterpart in (9) because only active actuators are taken into account and, therefore, requires less computational cost. Moreover, solving multiple optimization problems subsequently requires less computational cost compared to solving all the problems at once [as in (9)] since the complexity of a QCQP or QP does not scale linearly with the number of decision variables [15].

\section{Experimental CASE Study}

In this section, we present an experimental study on the industrial IPMLSM-based transportation system presented in Fig. 1. In particular, we show the performance 
improvements of the proposed control allocation strategy, compared to the state-of-practice control solution currently applied by the manufacturer. In this experimental study, we show the unconstrained, distributed allocation procedure, as discussed in Section III-C, to illustrate the achievable performance improvement. The constrained allocation procedure has been illustrated by a simulation study on the same system in [13].

The considered system consists of two carriers and six actuators (see Fig. 1) and is modeled by (1)-(3) with $n=2$ and $m=6$. The carrier mass is $10.8 \mathrm{~kg}$. The system is driven by an industrial precision motion control system (NYCe 4000), developed by the same manufacturer. All experiments are carried out at a sampling rate of $4 \mathrm{kHz}$, and, using the decentralized approach in Section III-C, the allocation problem can be solved well within each sampling interval. The actuators are spaced such that the center distance between subsequent actuators is equal to the carrier length (see Fig. 3), unless specifically stated otherwise. Both the state-of-practice control strategy and the allocation strategy use a dedicated high-level (loop-shaped) motion controller for each carrier separately. We use the same stabilizing motion controller (identical to the one designed by the manufacturer) for both carriers, consisting of a feedback term (lead filter, integrator, and a low-pass filter), and a feedforward term. The feedback term for each carrier in the Laplace domain is given by

$$
\begin{aligned}
& \tau_{f b, j}(s) \\
& \quad=K \frac{1500 s^{2}+1 \cdot 10^{5} s+1.58 \cdot 10^{6}}{1.1 \cdot 10^{-4} s^{4}+0.23 s^{3}+236.7 s^{2}+6.28 \cdot 10^{4} s} e_{j}(s)
\end{aligned}
$$

with $s \in \mathbb{C}, e_{j}=r_{j}-y_{j}$ the position error signal of carrier $j$, and $r_{j}$ the position reference of carrier $j$. The stabilizing feedback controller achieves the specified crossover frequency of $20 \mathrm{~Hz}$ and satisfies standard robustness margins. The feedforward term consists of an acceleration feedforward term, a Coulomb friction compensation term, and an experimentally obtained term $F_{\delta, j}$ that compensates for magnet interaction forces between carriers, i.e.,

$$
\tau_{f f, j}=0.9 M_{j} \ddot{r}_{j}+K_{F} \operatorname{sign}\left(\dot{r}_{j}\right)+F_{\delta, j} .
$$

Although the Coulomb friction in the system slightly varies over time and may be position dependent, the experimentally validated value $K_{F}=4.9 \mathrm{~N}$ is chosen such that no overcompensation of Coulomb friction takes place but still compensates for a significant part of it. The total desired control force on carrier $j$ is then given by $\tau_{c, j}=\tau_{f f, j}+\tau_{f b, j}$. Both the stateof-practice controller as the proposed allocation scheme aim at implementing the desired control force $\tau_{c, j}$ for each carrier in a different manner (leading to a different performance) as we will illustrate below.

\section{A. State-of-practice Control Strategy}

The state-of-practice control strategy currently used by the manufacturer operates in the $d q 0$ reference frame (see Fig. 2) so that $i_{q}$ is the only control input for each carrier (for the considered control system, the direct current $i_{d}$ is controlled to zero). The force-generating component $i_{q}$ is then obtained by dividing the desired control force $\tau_{c}$ by a fixed

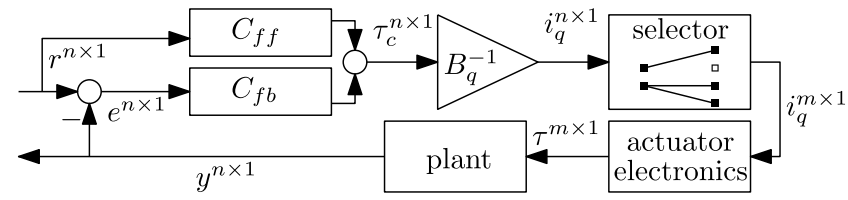

Fig. 6. State-of-practice control strategy. $C_{f b}$ and $C_{f f}$ indicate the feedback and feedforward parts of the motion controller, respectively. The superscripts on the signals indicate their dimensions.

motor gain $B_{q}=23.565$ N/A, see Fig. 6. By employing a strict actuator spacing, actuator end-effects are balanced so that the sum of the motor gains of the active actuators is approximately constant, regardless of the carrier position on the tracks. This control signal is then applied to each actuator that commutes with the considered carrier by means of a selector. However, when an actuator influences two carriers, the selector implements the control signal for the carrier that overlaps the actuator the most.

If an actuator commutes with only a single carrier, then this control strategy works well, as the actuator indeed applies the desired control force on the carrier by applying the correct commutation angle (which is a function of the carrier position). This control strategy, however, does no longer work properly when an actuator influences two carriers. The correct commutation angle is then only applied for the most overlapping carrier, which results in a wrong commutation angle for the second carrier. As a result, there is a mismatch between the desired and implemented control forces on the carrier that overlaps the actuator the least, compromising tracking performance. The implications of this fact are illustrated by the experimental results in Section IV-C below.

Remark 3: Although the state-of-practice control strategy may be improved by manual compensation of the mentioned disturbance forces for simple system layouts, the proposed allocation technique offers a systematic and scalable approach for robustly improving tracking performance also suitable for large-scale systems with many carriers and actuators.

\section{B. Proposed Allocation Strategy}

In contrast to the state-of-practice control strategy, the allocation scheme is applied in the $\alpha, \beta$ reference frame. The coefficients of the position-dependent motor gain matrix $B(y)$ in (3) and (6) are obtained from FEM-based electromagnetic simulations (see [1]) by measuring the relative position $z_{j k}$ of carrier $j$ with respect to actuator $k$. We assume that all actuators are identical, see Assumption 2. The motor gains, as a function of $z_{j k}$, are presented in Fig. 7, where the deterioration of the gains at both ends of the region of influence can be observed (i.e., the end-effects). The weighting matrices are $Q=10^{4} I$ and $W=I$, with $I$ the identity matrix of appropriate dimensions.

\section{Comparative Performance Study}

We have implemented both the state-of-practice and allocation control strategies on the experimental setup of Fig. 1. The following scenarios are studied: a) tracking of a demanding motion profile for a single carrier, with strict actuator spacing; b) tracking of a motion profile for a single carrier, with relaxed actuator spacing; c) a parallel motion of two carriers; 


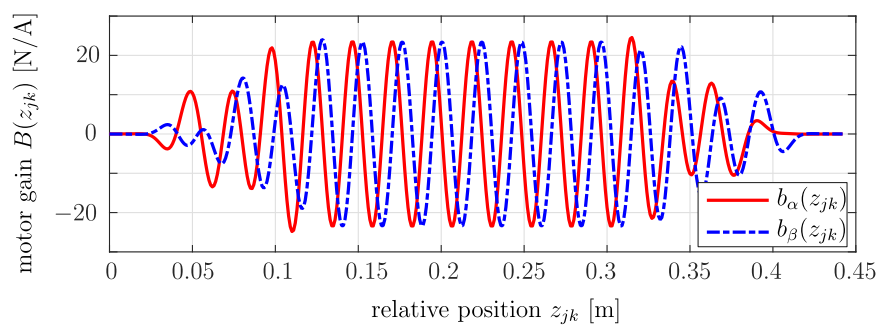

Fig. 7. Motor gains as a function of the relative position.

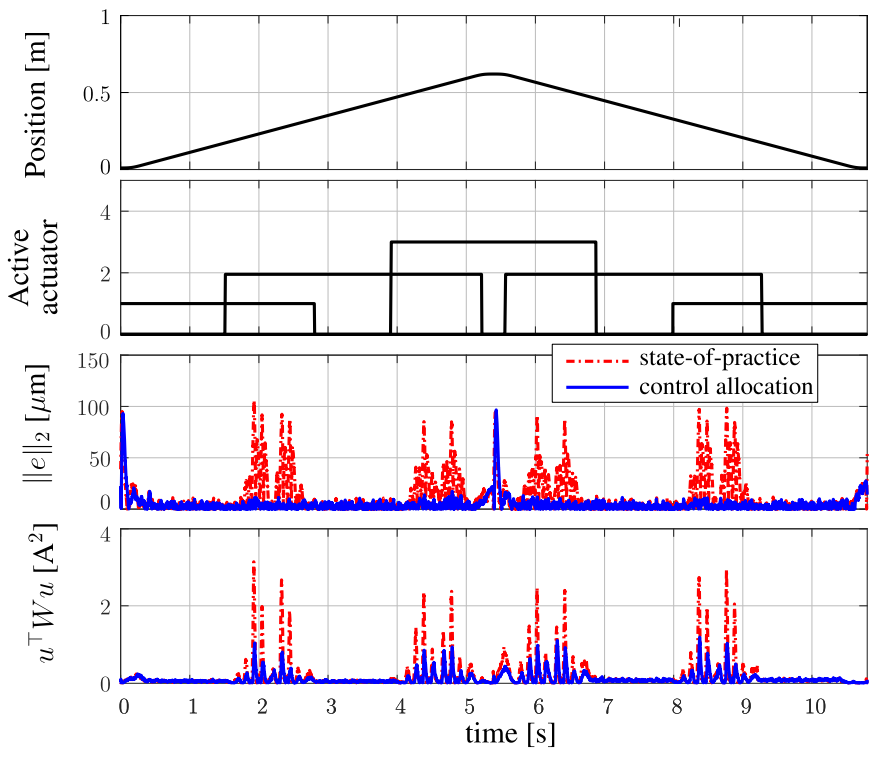

Fig. 8. Experimental results for Scenario b.

and d) a complex motion of two carriers, combining independent and adjacent motion. The results for Scenarios $b-d$ are presented in Figs. 8-10. In these figures, four subplots are presented which show the following: the top subplot shows the reference trajectories of the carriers, and the second subplot shows the active actuators at each time instant. Herein, the time intervals where an actuator influences two carriers are indicated by gray patches (in Figs. 9 and 10). The third subplot shows the Euclidian norm of the position error $\|e\|_{2}$ (with $e=\left[e_{1}, \ldots, e_{n}\right]^{\top}$ ), and the fourth subplot shows a metric for the power consumption, given by $u^{\top} W u$.

For Scenario $a$, both control strategies perform well during the constant velocity phase, despite the fact that multiple actuators influence the carrier. This is due to strict actuator spacing, that results in a smooth transition of a carrier between actuators (see Section II-A), and the fact that there is only one carrier on the tracks in this scenario.

Scenario b: Consider Fig. 8, which shows the experimental results of a single carrier performing a back and forth motion, where the actuator spacing has been relaxed. In particular, the second actuator has been shifted to the right by $6 \mathrm{~mm}$. A strict actuator spacing realizes that the sum of the motor gains of the active actuators is constant, regardless of the carrier position on the tracks. In the current scenario, this property is violated by the relaxed actuator spacing, and, as a result, the state-of-practice control strategy implements a force that deviates from the desired control force coming from the motion controller. As can be observed in the third subplot of
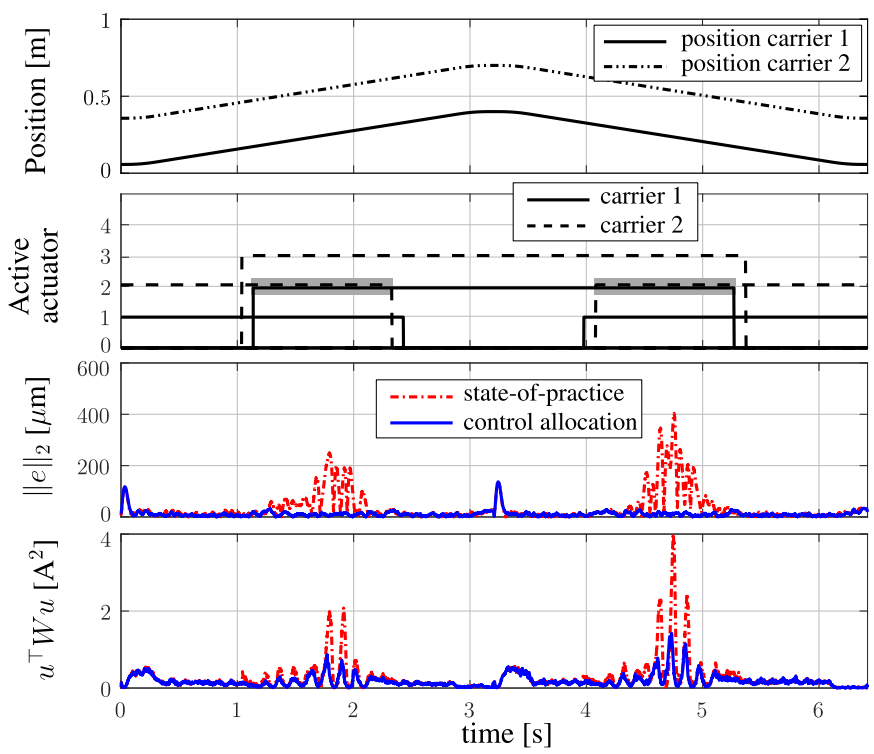

Fig. 9. Experimental results for Scenario c.

Fig. 8, this results in increased position errors in the regions where multiple actuators are active. The proposed allocation scheme is instead able to adapt and realizes a low tracking error while minimizing power consumption. The increased position error at the start of the experiment and velocity reversals is due to any remaining Coulomb friction.

Scenario c: For this experiment, the strict actuator spacing has been restored. Consider Fig. 9. Two carriers perform an adjacent back and forth motion, with two time intervals at which actuator 2 influences both the carriers. In these intervals, the state-of-practice controller results in a large position error, which is caused by the fact that the shared actuators are only able to take the correct commutation angle for the most overlapping carrier. The other carrier then experiences a control force that deviates from the desired control force coming from the motion controller, due to a mismatch in the correct and attained commutation angles. The proposed allocation scheme is instead able to adapt and finds a control input such that the trajectory can be followed well with significantly reduced position errors. Moreover, as the bottom subplot indicates, the power usage is decreased as well.

Scenario d: Consider Fig. 10. In this experiment, two carriers perform a complex motion. The carriers move independently as well as adjacent, where actuator 2 influences both carriers during the majority of the experiment. As discussed in Scenario c, the actuator can only take the correct commutation angle for the most overlapping carrier when using the state-of-practice control strategy, which results in pronounced large position errors. The proposed allocation scheme is instead capable of adapting to such a situation by altering the current inputs, resulting in low position errors. This shows the capability of the system to perform independent motion tasks with high accuracy. During the adjacent motion (in the time interval 2.7-5.8 s), the system behaves similar to Scenario $\mathrm{c}$, where the allocation scheme indeed outperforms the state-of-practice control solution in terms of position accuracy, see the inset in the third subplot. Note that the left and right error peaks in the inset are due to friction. 


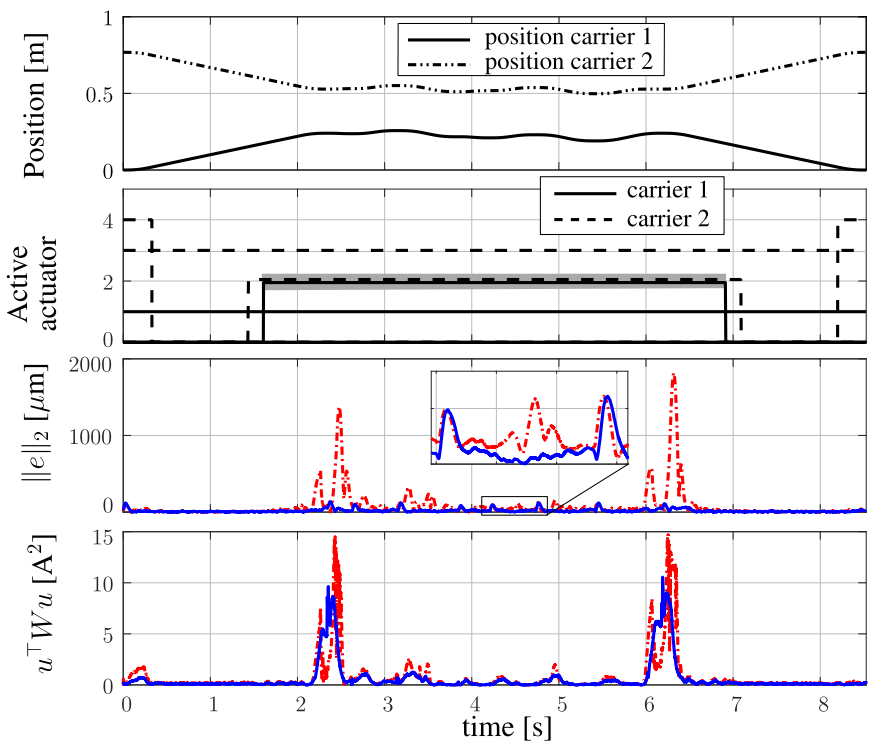

Fig. 10. Experimental results for Scenario d.
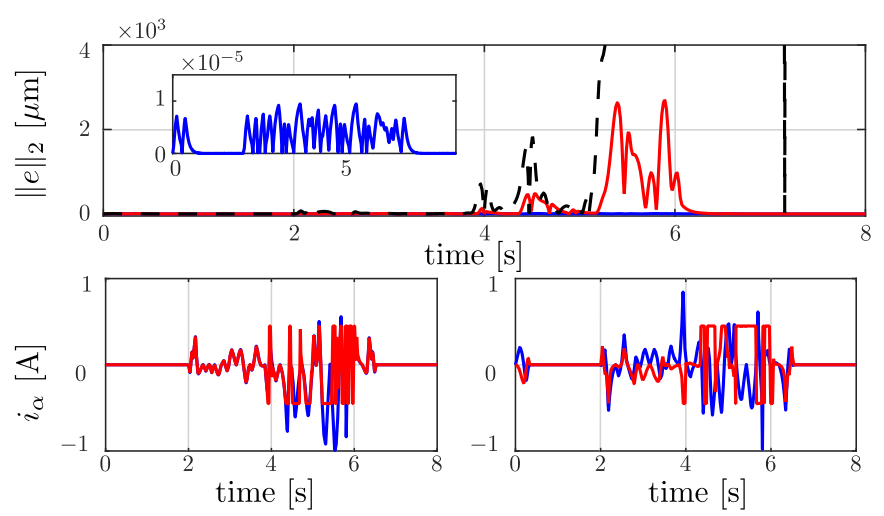

Fig. 11. Simulation results for Scenario d, for the state-of-the-practice controller (black dashed line), unconstrained control allocation (blue line), and constrained control allocation scheme (red line). The bottom plots show the $\alpha$-currents for two actuators.

\section{Discussion}

The experimental study shows that the proposed control allocation scheme (compared to the state-of-practice controller used by the manufacturer) achieves improved tracking performance, evident from the root mean square (rms) values of the norm of the error $\|e\|_{2}$ for Scenarios a-d, presented in the following table:

\begin{tabular}{lll|lll}
\hline scenario & state-of-pr. & alloc. & scenario & state-of-pr. & alloc. \\
\hline $\mathrm{a}$ & 0.0507 & 0.0111 & $\mathrm{c}$ & 0.0681 & 0.0185 \\
$\mathrm{~b}$ & 0.0230 & 0.0097 & $\mathrm{~d}$ & 0.2193 & 0.0291 \\
\hline
\end{tabular}

Furthermore, the allocation scheme provides the possibility to take actuator limits into account. This is illustrated by a simulation study of Scenario d, where $i_{\alpha}$ and $i_{\beta}$ are limited to a challenging value of $0.4 \mathrm{~A}$. The results in Fig. 11 illustrate that employing the constrained control allocation scheme results in decreased tracking performance, compared to the unconstrained case, but realizes this performance with significantly less actuator duty. On the other hand, the constrained allocation approach clearly outperforms the state-of-practice controller, which results in very large positioning errors.
For more simulation results concerning the constrained case, see [13].

\section{CONCLUSION}

We have presented a control allocation framework for an industrial high-accuracy transportation and positioning system, which results in enhanced tracking, allows for independent motion of multiple carriers, is able to take actuator limits into account, and results in relaxed hardware design specifications. A decentralized allocation procedure is presented that enhances computational efficiency, such that the control allocation scheme is suitable for online implementation at high sampling rates, and facilitates scalability to larger system configurations. We have illustrated the benefits of the proposed control allocation strategy by means of an experimental study.

\section{REFERENCES}

[1] J. M. M. Rovers, J. W. Jansen, and E. A. Lomonova, "Novel force ripple eduction method for a moving-magnet linear synchronous motor with a segmented stator," in Proc. Int. Conf. Elect. Mach. Syst., Oct. 2008, pp. 2942-2947.

[2] B. Rexroth. Industrial Applications of an IPMLSM. Accessed: Jun. 1, 2019. [Online]. Available: http://www.boschrexroth.com/lms

[3] I. Boldea and S. A. Nasar, Vector Control of AC Drives. Orlando, FL, USA: CRC Press, 1992.

[4] J. F. Gieras, Z. J. Piech, and B. Tomczuk, Linear Synchronous Motors, 2nd ed. Boca Raton, FL, USA: CRC Press, 2012.

[5] H. W. Beaty and J. Kirtley, Electric Motor Handbook. New York, NY, USA: McGraw-Hill, 1998.

[6] K. Zhou, J. C. Doyle, and K. Glover, Robust and Optimal Control. Upper Saddle River, NJ, USA: Prentice-Hall, 1996.

[7] O. Härkegård and S. T. Glad, "Resolving actuator redundancy—Optimal control vs. control allocation," Automatica, vol. 41, no. 1, pp. 137-144, 2005.

[8] T. A. Johansen and T. I. Fossen, "Control allocation-A survey," Automatica, vol. 49, no. 5, pp. 1087-1103, 2013.

[9] D. Q. Mayne, "Model predictive control: Recent developments and future promise," Automatica, vol. 50, no. 12, pp. 2967-2986, Dec. 2014.

[10] G. F. Franklin, J. D. Powell, and A. Emami-Naeini, Feedback Control of Dynamic Systems, 7th ed. Boston, MA, USA: Pearson, 2015.

[11] Y. Chen and J. Wang, "Adaptive energy-efficient control allocation for planar motion control of over-actuated electric ground vehicles," IEEE Trans. Control Syst. Technol., vol. 22, no. 4, pp. 1362-1373, Jul. 2014.

[12] A. A. Mamun, I. Mareels, T. H. Lee, and A. Tay, "Dual stage actuator control in hard disk drive-A review," in Proc. 29th Annu. Conf. IEEE Ind. Electron. Soc. (IECON), Nov. 2003, pp. 2132-2137.

[13] R. Beerens et al., "Control allocation for a high-precision linear transport system," in Proc. 57th IEEE Conf. Decis. Control (CDC), Dec. 2018, pp. 1657-1662.

[14] H. A. Toliyat and S. G. Campbell, DSP-Based Electromechanical Motion Control. Boca Raton, FL, USA: CRC Press, 2003.

[15] S. Boyd and L. Vandenberghe, Convex Optimization. New York, NY, USA: Cambridge Univ. Press, 2004.

[16] K. A. Bordingnon and W. C. Durham, "Closed-form solutions to constrained control allocation problem," J. Guid., Control, Dyn., vol. 18 , no. 5, pp. 1000-1007, 1995.

[17] CPLEX Optimizer. Accessed: Jun. 1, 2019. [Online]. Available: https://www.ibm.com/analytics/cplex-optimizer

[18] Gurobi Optimization. Accessed: Jun. 1, 2019. [Online]. Available: https://www.gurobi.com/

[19] J. Nocedal and S. Wright, Numerical Optimization, 2nd ed. New York, NY, USA: Springer, 2006.

[20] O. Härkegåkd, "Efficient active set algorithms for solving constrained least squares problems in aircraft control allocation," in Proc. 41st IEEE Conf. Decis. Control (CDC), vol. 2, Dec. 2002, pp. 1295-1300.

[21] J. A. M. Petersen and M. Bodson, "Interior-point algorithms for control allocation," J. Guid., Control, Dyn., vol. 28, no. 3, pp. 471-480, 2005.

[22] G. Chartrand, Introductory Graph Theory. New York, NY, USA: Dover, 1985.

[23] S. Even, Graph Algorithms, 2nd ed. New York, NY, USA: Cambridge Univ. Press, 2012. 